\title{
Aural CT characteristics of American Cocker Spaniels have features of both mesaticephalic and brachycephalic breeds
}

Kaimio, Mirja L. M.

2020-11

Kaimio , M L M , Lappalainen , A K, Rahmani , V , Männikkö , S \& Laitinen-Vapaavuori , O M 2020 , ' Aural CT characteristics of American Cocker Spaniels have features of both mesaticephalic and brachycephalic breeds ' , Veterinary Radiology \& Ultrasound , vol. 61 , no. 6 , pp. 649-658 . https://doi.org/10.1111/vru.12900

http://hdl.handle.net/10138/333216

https://doi.org/10.1111/vru.12900

acceptedVersion

Downloaded from Helda, University of Helsinki institutional repository.

This is an electronic reprint of the original article.

This reprint may differ from the original in pagination and typographic detail.

Please cite the original version. 
1 Aural computed tomography characteristics of American cocker spaniels have features of both mesaticephalic and brachycephalic breeds.

3

4 Authors: Mirja L. M. Kaimio, ${ }^{1}$ Anu K. Lappalainen, ${ }^{1}$ Vahideh Rahmani, ${ }^{1}$ Sofia Männikkö, ${ }^{2}$ Outi 5

M. Laitinen-Vapaavuori ${ }^{1}$

Corresponding author: Mirja Kaimio, Faculty of Veterinary Medicine, P.O. Box 57,

00014 University of Helsinki, Finland, mirja.kaimio@evidensia.fi

Key words: otitis externa, canine, middle ear, otitis media

Conflicts of interest: The authors state no conflict of interest.

16 None of the findings of this study have been presented or published previously elsewhere.

EQUATOR Network disclosure: An EQUATOR network checklist was not used.

18 Abbreviations:

19 TA: transverse area

20 TA1: transverse area of the osseous horizontal ear canal

21 TA2: transverse area of the widest air-filled part of the cartilaginous horizontal ear canal 
Otitis externa and otitis media are common in American cocker spaniels, however breed-specific aural computed tomography (CT) descriptions are currently lacking. This prospective and retrospective, observational, analytical study aimed to describe quantitative CT characteristics of the horizontal ear canal and the tympanic bulla in American cocker spaniels versus similar-sized mesaticephalic dogs. We prospectively performed clinical examinations and aural CT scanning for 38 American cocker spaniels. Computed tomographic transverse area of the osseous (TA1), the widest air-filled part of the cartilaginous (TA2) horizontal ear canal, tympanic bulla volume and tympanic bulla wall thickness were measured. The TA1 and TA2 measurements were compared with those of 23 retrospectively recruited, similar-sized mesaticephalic dogs that had undergone CT-scanning of the head for non-ear related reasons. No significant differences appeared in TA1 and TA2 between healthy American cocker spaniels and mesaticephalic dogs, but severely affected American cocker spaniels had significantly smaller TA2 $(P<0.001)$. The intraclass correlation coefficient for intra-observer and inter-observer repeatability was 0.972 and 0.983 for TA1 and 0.994 and 0.998 for TA2, respectively. Variation between individuals was subjectively noted in healthy and affected American cocker spaniels, but the mean tympanic bulla volume was slightly smaller in relation to the body weight, and the tympanic bulla wall was thicker than in previous reports for mesaticephalic dogs. The tympanic bulla wall appeared thicker rostro-ventrally than caudo-ventrally in $44 \%$ of the dogs. Our results imply that a relatively thick tympanic bulla wall may be a normal CT variation and should be interpreted cautiously in this breed. 
Cocker spaniels are reported to be overrepresented among breeds that suffer

51 from otitis externa, ${ }^{1-4}$ one of the most common dermatological conditions in dogs. ${ }^{5}$ In a recent survey, the prevalence of otitis externa in American cocker spaniels was $27 \%{ }^{6}$ Accordingly, they are also overrepresented among breeds requiring total ear canal and bulla osteotomy surgery as a treatment for chronic otitis externa. ${ }^{7-10}$ Otitis media accompanies chronic otitis externa in up to $80 \%$ of the cases. ${ }^{11}$ However, the diagnosis of otitis media is often difficult, and diagnostic imaging can be used to aid the diagnosis. ${ }^{12}$ Computed tomography (CT) and magnetic resonance imaging (MRI) are more sensitive than traditional radiography or ultrasonography in diagnosing otitis media. ${ }^{13-18}$ Currently, CT is considered the gold standard method to evaluate the tympanic bulla. ${ }^{19,20}$ While CT is becoming increasingly available in veterinary practice, it is often used to evaluate the pathological changes caused by chronic otitis, especially when assessing whether surgery is required. ${ }^{21}$ of the tympanic bulla volume and wall, as well as tympanic bulla shape and position, have been described in mesaticephalic and brachycephalic dogs. ${ }^{23-25}$ Previous studies have shown that brachycephalic breeds have a smaller tympanic bulla volume and a thicker bulla wall than mesaticephalic breeds, and that there is interbreed variation in the shape and position of the tympanic bulla. For example, Cavalier King Charles spaniels appear to have a flattened tympanic bulla, ${ }^{25}$ and English bulldogs have a narrower horizontal ear canal than mesaticephalic breeds. ${ }^{26}$ However, breed-specific aural CT measurements are lacking in non71 brachycephalic breeds with high-risk for otitis externa, such as American cocker spaniels, but 72 would be beneficial in the diagnostics of ear diseases in these breeds. 
74 horizontal ear canal and the tympanic bulla in American cocker spaniels with healthy and

diseased ear canals. Another aim was to evaluate differences in the transverse area (TA) measurements of the horizontal ear canal between healthy American cocker spaniels and other mesaticephalic dog breeds, and among American cocker spaniels of different earhealth conditions. In addition, this study aimed to investigate the intra-observer and interobserver repeatability of the TA measurements. Our hypothesis was that the horizontal ear canal in American cocker spaniels is narrower than in a group of similar-sized mesaticephalic dogs and that the repeatability of the measurements within and between observers is high.

\section{MATERIALS AND METHODS}

\section{Case selection}

This study was a prospective and retrospective, observational, analytical design. All procedures were approved by the Finnish National Animal Experiment Board (ESAVI-1662/04.10.03/2011). The prospective part of the study was carried out at the Veterinary Teaching Hospital of the University of Helsinki during the period of march to september 2011. All owners signed an informed consent before entering the study. One of the authors (a senior veterinary clinician, M.K.) evaluated each participating dog to match the inclusion criteria: pure-bred American cocker spaniel, aged at least 12 months with or without a history of otitis externa, and no known conditions that increase the risk of complications from anesthesia. All animals meeting inclusion criteria during a 6-month study recruitment period were included in the study.

Clinical examination 
97 (M.K.). The dogs were sedated intramuscularly with medetomidine $10 \mathrm{ug} / \mathrm{kg}$ (Domitor ${ }^{\circledR}$, Orion

Pharma, Espoo, Finland) and butorphanol $1 \mathrm{mg} / \mathrm{kg}$ (Torbugesic ${ }^{\circledast}$, Forte-Dodge, lowa, USA), and

their ears were examined with a video-otoscope (Dr Fritz GmbH, Tuttlingen, Germany). The

100 presence or absence of otitis externa was based on the OTIS3 score as described by Nuttal et

101 al. (2014). ${ }^{27}$ A score between 0-12 was assigned to each ear, and a total ear score of $\geq 4$

102 differentiated affected from clinically healthy ears. In addition, samples for cytology were

103 taken from each ear. Cytology slides were prepared and examined under oil immersion (OI, $\mathrm{x}$

104 1000) as described previously. ${ }^{4}$

105 Computed tomography imaging

Following clinical examination, CT-scanning was performed on the ears using a

107 helical dual slice scanner (Somatom Emotion Duo; Siemens AG, Forchheim, Germany) with

$108130 \mathrm{kVp}, 113 \mathrm{~mA}$, with a bone algorithm. The slice thickness was $1 \mathrm{~mm}$, feed/rotation $2 \mathrm{~mm}$,

109 and reconstruction increment $0.5 \mathrm{~mm}$. The dogs were placed in a sternal recumbency and

110 their head was supported straight. They received midazolam $0.1 \mathrm{mg} / \mathrm{kg}$ IV (Midazolam

111 Hameln, Siegfried Hameln $\mathrm{GmbH}$, Hameln, Germany) if more sedation was needed. The

112 images were stored in DICOM format in the hospital's PACS until analysis.

To compare the horizontal ear canal measurements between American cocker

114 spaniels and mesaticephalic dogs, the database of Veterinary Teaching Hospital of University

115 of Helsinki was searched for dogs that matched the prospectively sampled animals as much

116 as possible and had undergone CT-scanning of the head for reasons unrelated to ear disease.

117 The inclusion criteria for these dogs were: mesaticephalic breed weighing 5-20 kg, aged at 
118 least one year. Dogs were excluded if there was any history or clinical findings of ear or skin

119 disease in the database, if the slice thickness of the scan was more than $2 \mathrm{~mm}$, or if there

120 were incidental abnormalities (for example otolithiasis) in the CT images. A veterinary

121 radiology specialist (A.K.L., 15 years of experience in veterinary radiology) reviewed the CT

122 images of all retrieved cases to ensure that the inclusion criteria were fulfilled.

123 Image analysis

Image evaluations were performed by the veterinary radiology specialist

125 (A.K.L.) and two licensed veterinarians (observer A: M.K., and observer B: V.R.). All CT

126 studies were viewed using commercially available software (OsiriX MD, version 9.0, OsiriX

127 Foundation, Geneva, Switzerland). In American cocker spaniels, the following parameters

128 were recorded from the CT images: 1$)$ transverse surface area $\left(\mathrm{mm}^{2}\right)$ of the osseous part of

129 the horizontal ear canal (TA1), 2) transverse surface area $\left(\mathrm{mm}^{2}\right)$ of the widest air-filled part

130 of the cartilaginous horizontal ear canal (TA2), 3) thickness (mm) of the tympanic bulla wall,

131 4) tympanic bulla volume $\left.\left(\mathrm{mm}^{3}\right), 5\right)$ presence of fluid/soft tissue material in the tympanic

132 bulla, subjectively ranked as mild $(<30 \%)$, moderate $(30-60 \%)$ or marked $(>60 \%), 6)$

133 presence of ear canal mineralization, subjectively ranked as mild $(<30 \%)$, moderate

134 (30-60\%) or marked (>60\%), 7) skull width $(\mathrm{cm})$, and 8) skull height $(\mathrm{cm})$. In mesaticephalic

135 dogs, the horizontal ear canal measurements (TA1 and TA2), and the skull measurements

136 (width and height) were performed.

138 for the measurements of the TA $\left(\mathrm{mm}^{2}\right)$ of the horizontal ear canal. The window level and

139 width were $300 \mathrm{HU}$ and $1500 \mathrm{HU}$, respectively, following Seppänen et al. (2019). ${ }^{26}$ First, the 
140 dorsal image plane was determined from the midsagittal image as a parallel plane to the

141 skull base (Figure 1A). Second, the transverse image was straightened at the level of the

142 middle ears using the cochleae as landmarks. The parasagittal image for measuring the TA of

143 the osseous ear canal (TA1) was the one in which the bony wall was most clearly seen

144 (Figure 1B). The oblique parasagittal image for measuring the TA of the widest air-filled part

145 of the cartilaginous horizontal ear canal (TA2) was selected as the one in which the cross-

146 section of the canal was considered the largest (Figure 1C). The images were randomized,

147 and the observers were blinded to the background data of the dogs. Before all

148 measurements, observers A and B familiarized themselves with the measurement

149 techniques using practice images under the supervision of the veterinary radiology specialist.

150 The TA was determined by manually drawing a region of interest with the software's pencil

151 tool, tracing the margin of the bony wall of the osseous (TA1) and the widest air-filled (TA2)

152 cartilaginous horizontal ear canal (Figure 2). Observers A and B performed these

153 measurements twice, one week apart, for intra-observer and inter-observer repeatability

154 testing. Finally, the mean of the four TA1 and TA2 measurements $\left(\mathrm{mm}^{2}\right)$ in each dog was

155 calculated and used for the study.

Observer A measured the thickness of the tympanic bulla wall. It was measured

157 at its most ventral point parallel to the midline in the transverse plane images (middle-ear

158 algorithm, window level $700 \mathrm{HU}$, window width $4000 \mathrm{HU}$ ) at two levels - at the level of the

159 tympanic membrane (caudo-ventral aspect) and at the level of the cochlea (rostro-ventral

160 aspect) (Figure 3). Observer $B$ measured the tympanic bulla volume using the same image

161 parameters. It was measured by calculating all the sequential hand-drawn regions of interest

162 together using the computer program. The internal surface of the osseous bulla including 
the three middle ear bones was manually traced in all slices. In the sections located at the

164 level of the tympanum and where the tympanic membrane was not visible, a straight line

165 was traced artificially to connect the medial edges of the horizontal ear canal by using a 45-

166 degree angle in relation to the central axis of the canal. After tracing the region of interest in

167 all slices of each ear, the total volume of each middle ear was calculated automatically by

168 the software. In addition, to compare the horizontal ear canal measurements with the skull

169 size, observer A measured the skull width and height from the transverse image of the skull

170 at the same multiplanar reconstruction level and window settings as the tympanic bulla

171 caudo-ventral measurements. The skull width was measured as the widest part of the skull

172 dorsal to the cochleae in the horizontal plane; the skull height was the measurement from

173 the base of the skull to the top of the skull in the midline (Figure 4). The skull width and

174 height were calculated together to describe the skull size.

175 Statistical analysis

The American cocker spaniels were divided into four groups according to the

177 history of otitis externa and the total ear score: clinically healthy ears (score 0-3 in both ears)

178 with no history of ear disease (group 1), clinically healthy ears (score 0-3 in both ears) with a

179 history of ear disease (group 2), mild otitis externa (score 4-5 in either or both ears, group 3)

180 and severe otitis externa (score $\geq 6$ in either or both ears, group 4).

A statistician (S.M.) performed all statistical analyses using statistics software

182 (SAS ${ }^{\circledR}$ System for Windows, version 9.4, SAS Institute Inc., Cary, NC, USA). For investigating

183 differences in TA1 and TA2 between healthy American cocker spaniels (group 1) and

184 mesaticephalic dogs, a random effects ANCOVA model was fitted with a fixed effect of breed

185 group, covariates of age, weight, gender, and skull sum, and a random effect of dog. TA1, 
186 TA2, the tympanic bulla wall thickness (rostral and caudal), and the tympanic bulla volume

187 were also compared between the four American cocker spaniel groups. These endpoints

188 were modelled as described above, except the American cocker spaniel group was included

189 in the model instead of the breed group. In addition, in American cocker spaniels,

190 associations between horizontal ear canal edema or exudate and TA2-values were

191 investigated with one-way ANOVA models. Associations between different aural cytological

192 findings or the presence of ear canal mineralization and the presence of tympanic bulla

193 effusion were assessed with mixed effects logistic regression models. Dog was used as a

194 random effect in both, the ANOVA and the logistic regression models.

In the TA1 and TA2 measurements, the intraclass correlation coefficient (ICC)

196 was calculated both for intra-observer and inter-observer comparisons. For inter-observer

197 calculations the mean of the two measurements was used for each assessor. In addition to

198 ICC, a random effects model was fitted separately for both comparisons to estimate the

199 variance components related to repeating the assessment by the same observer (intra-

200 observer comparison), or the variance component related to the observer (inter-observer

201 comparison). Both models included dog as a random effect. Values of ICC $0.01-0.2$ were

202 considered to have "slight agreement", 0.21-0.40 "fair agreement", 0.41-0.60 "moderate

203 agreement", 0.61-0.80 "substantial agreement", and 0.80-1.00 "almost perfect

204 agreement". ${ }^{28}$ Intra-observer and inter-observer coefficients of variation for TA1 and TA2

205 were calculated using the root mean square method.

For ANCOVA models, estimates of group differences and within-group values

207 were calculated from the fitted models with contrasts. For the group differences and within-

208 group values, $95 \%$ confidence intervals $(\mathrm{Cl})$ and $P$-values were calculated. For the multiple

209 comparisons between American cocker spaniel groups, the $P$-values were adjusted with the 
210 Tukey-Kramer method. Age, weight, and skull sum were tested with T-tests for comparison

211 between healthy American cocker spaniels and mesaticephalic dogs. For comparisons among

212 the American cocker spaniel groups, a one-way ANOVA was used for these variables.

213 Differences between groups in the proportion of each gender were estimated with Fisher's

214 exact test. Normality assumptions for parametric models were confirmed with Kolmogorov-

215 Smirnov tests, for borderline cases also the skewness parameter was assessed. $P$-values $<$

2160.05 were considered statistically significant.

Additionally, it was evaluated whether the measured tympanic bulla volume

218 followed the previously published equation for middle ear canal volume (MECV) $=-0.612+$

$2190.757 * \ln$ (bodyweight). ${ }^{23}$ The MECV values were calculated for all dogs based on the

220 equation, and compared with the measured tympanic bulla volume values. To describe the

221 difference, a Bland-Altman plot was created. Bland-Altman plot presented the difference

222 between MECV and measured tympanic bulla volume values against the mean of these two.

RESULTS

225 Study population

Thirty-eight (26 females, 12 males) privately owned American cocker spaniels

227 met the inclusion criteria and were enrolled in the study. The database search found 23 dogs

228 (12 females, 11 males) representing 18 different breeds to be included in the retrospective

229 part of the study. Most of these dogs $(n=21)$ had been imaged with the same scanner as the

230 American cocker spaniels in this study; the remaining two were scanned with a helical 64-

231 slice scanner (Lightspeed VCT, GE Healthcare, Wisconsin, USA). 
considered clinically healthy in both ears and were assigned to either group 1 ( 8 females, 1 male) or to group 2 ( 4 females, 5 males). Group 3 comprised seven ( 6 females, 1 male) dogs

with unilateral $(n=1)$ or bilateral $(n=6)$ mild otitis externa, and group 4, 13 (8 females, 5 males) dogs with unilateral $(n=4)$ or bilateral $(n=9)$ severe otitis externa. One dog with unilateral severe otitis externa had earlier total ear canal ablation and bulla osteotomy surgery performed on the other ear, so a total of 75 ears were examined. OTIS3 scoring and aural cytology results are shown in Table 1.

Measurements of the horizontal ear canal

In American cocker spaniels' group 1 and in mesaticephalic dogs, the mean TA1 was $27.2 \mathrm{~mm}^{2}$ and $29.2 \mathrm{~mm}^{2}$, whereas the mean TA2 was $37.1 \mathrm{~mm}^{2}$ and $35.6 \mathrm{~mm}^{2}$, respectively. TA1 and TA2 showed no significant differences between these two groups (Table 2). Significant differences were detected in age $(P=0.042)$ and gender $(P=0.050)$. In American cocker spaniels (groups 1-4), the mean TA1 showed no significant differences between the four groups (Figure 5). In the analysis of TA2, a significant group effect was found $(P<0.001)$. TA2 values were significantly lower in group 4 than in the other groups. (Figure 6). Marked edema $(P<0.001)$ and moderate or marked amount of exudate $(P<0.001)$ in the horizontal ear canal all significantly diminished TA2.

Repeatability in TA1 and TA2 measurements showed almost perfect agreement within and between observers. For observer A, ICC was 0.959 for TA1 and 0.990 for TA2; for observer B, ICC was 0.985 for TA1 and 0.998 for TA2. Overall ICC-values for the intraobserver and inter-observer repeatability were 0.972 and 0.983 for TA1, and 0.994 and 0.998 for TA2, respectively. Intra-observer and inter-observer coefficients of variation were $3.2 \%$ and $2.3 \%$ for TA1 and $3.7 \%$ and $2.0 \%$ for TA2, respectively. 
in group 1, 1/18 (6\%) ears in group 2, 4/14 (29\%) ears in group 3 and 17/25 (68\%) ears in

259 group 4 had evidence of ear canal mineralization. The mineralization was considered mild in

26016 ears (groups 2-4), moderate in 2 (group 4), and severe in 4 (group 4) ears.

261 Measurements of the tympanic bulla

In group 1, the mean tympanic bulla volume was $0.77 \mathrm{~mm}^{3}$ and the tympanic

263 bulla wall thickness varied between $0.7 \mathrm{~mm}$ and $2.7 \mathrm{~mm}$ (Table 3, Figures 3 and 7). The mean

264 thickness of the tympanic bulla wall was $1.3 \mathrm{~mm}$ at the level of the tympanic membrane, and

$1.6 \mathrm{~mm}$ at the level of the cochlea. In 33 of 75 (44\%) ears (groups 1-4), the thickness of the

bulla wall was at least $0.3 \mathrm{~mm}$ thicker at the rostro-ventral measurement point. Groups 1-4

showed that the overall mean difference detected between the calculated MECV and the

measured tympanic bulla volume was $0.34 \mathrm{~mm}^{3}$ (Figure 8).

Six American cocker spaniels had fluid or soft tissue material in their bulla

271 either bilaterally $(n=5)$ or unilaterally $(n=1)$. The amount of material in the bulla was

272 considered mild in 2, moderate in 3 and marked in 6 ears. Nine out of 22 ears (41\%) with

273 clinically severe otitis externa showed material in the bulla. One dog with clinically mild otitis

274 externa in the left ear had a marked amount of material in the left bulla and a mild amount

275 of material in the right bulla; the right external ear was considered clinically healthy. The

276 presence of ear canal mineralization $(P=0.016)$, as well as the presence of cocci $(P=0.031)$

277 or rod-shaped bacteria $(P=0.026)$ in cytology of the aural exudate, were associated with the 278 presence of bulla effusion. 
282 the horizontal ear canal and the tympanic bulla in American cocker spaniels, a high-risk

283 breed for otitis externa. Findings indicated that the transverse area of both the osseous and

284 the cartilaginous part of the horizontal ear canal in clinically healthy American cocker

285 spaniels did not differ from mesaticephalic dogs, which was contradictory to our hypothesis.

286 It appears that, in comparison to other similarly sized breeds, an anatomically narrower

287 horizontal ear canal is not the explanation why otitis externa and end-stage ear disease are

288 overrepresented in American cocker spaniels. However, the tympanic bulla wall was thicker

289 and the tympanic bulla volume smaller in this breed than what has been previously

290 published for mesaticephalic dogs. These results indicated that breed-specific data may be

291 important when interpreting aural CT images.

Previous studies have shown that body weight correlates positively with the

overall length and diameter of the distal opening of the ear canal. ${ }^{29}$ Similarly, the volume of

294 the ear canal is dependent on body weight. ${ }^{30}$ However, anatomic conformation of the skull

295 also affects aural morphology. ${ }^{23-26}$ Our results showed that the size of the horizontal ear

296 canal lumen was similar in all the examined American cocker spaniels and mesaticephalic

297 dogs, with the mean TA of the osseous part of the horizontal ear canal being less than 30

$298 \mathrm{~mm}^{2}$. Taking into account the slight difference in methods, our measurement results of the

299 horizontal ear canal are in line with previous studies. ${ }^{26,29}$ Interestingly, a small and narrow

300 horizontal ear canal does not necessarily cause otitis externa, as most of the English bulldogs

301 with very narrow horizontal ear canals did not suffer from otitis. ${ }^{26}$ However, with

302 proliferative changes of the ear canal, the small lumen may readily disappear completely.

303 Compared to other breeds, American cocker spaniels present with ceruminous gland

304 hyperplasia and ectasia more often, which may lead to anatomical changes of the ear canal 
and occlusion of the ear canal lumen, resulting in end-stage otitis. ${ }^{10}$ Mineralization of the ear canal was very common in severely affected ears in this study, but previous studies have

307 reported mild ear canal mineralization to be common even in clinically healthy dogs. ${ }^{31}$

308 Notably, severe ear canal mineralization was ten times more common in our study than in 309 the previous study. ${ }^{31}$

$$
\text { We also evaluated the repeatability of the TA measurements within and }
$$

311 between observers. The method showed almost perfect agreement in the intra- and inter-

312 observer measurements. The variance components showed that the variation between dogs

313 was much larger than the variation between repeats and assessors. To further diminish the

314 impact of human measurement error in the future, automated measurement techniques

315 could be developed.

In American cocker spaniels, the tympanic bulla wall thickness varied

317 substantially between individuals within groups, including groups 1 and 2 . However, the

318 mean tympanic bulla wall thickness at the level of the tympanic membrane was $30 \%$ thicker

319 than what is previously reported for mesaticephalic dog breeds. ${ }^{24}$ Moreover, in the previous

320 study, ${ }^{24}$ the mean rostro-ventral measurements were similar to the caudo-ventral

321 measurements in mesaticephalic dogs. In our study, the tympanic bulla wall appeared

322 thicker rostro-ventrally in $44 \%$ of the American cocker spaniels. A thicker tympanic bulla wall

323 rostro-ventrally is typical for brachycephalic breeds. ${ }^{24}$ In our study, the difference was not

324 related to the positioning of the dogs, as all studied dogs were positioned in the same way.

325 In addition, the finding was bilateral - the left and the right bulla showed the same result in

326 clinically healthy American cocker spaniels. These results imply that the tympanic bulla wall

327 may be relatively thick also in some non-brachycephalic breeds due to anatomical variations

328 in the conformation. Moreover, the mean rostro-ventral measurement showed a thicker 
tympanic bulla wall at this level in both healthy and diseased American cocker spaniels,

330 indicating that the tympanic bulla anatomy in this breed is slightly different from

331 mesaticephalic breeds. Thus, in the absence of other abnormalities, a bilaterally symmetric

332 thick bulla wall should not be diagnosed as otitis media in this breed, as it may be a normal

333 variation.

The tympanic bulla volume also showed variation between individuals. The

mean tympanic bulla volume $\left(0.84 \mathrm{~mm}^{3}\right)$ was smaller than what is reported for

mesaticephalic breeds in general $\left(1.50 \mathrm{~mm}^{3}\right) \cdot{ }^{23}$ In relation to the body weight of the dog, the

tympanic bulla volume did not follow the previously reported MECV equation ${ }^{23}$ very well.

338 The study of Defalque et al. (2005) $)^{23}$ included only a few middle-sized (10 to $20 \mathrm{~kg}$ ) dogs,

339 which could explain the difference. Overall, the MECV equation seemed to give larger values

340 compared to the measured tympanic bulla volume. However, the bulla volume was in line

341 with the value previously reported for mesaticephalic dogs weighing less than $15 \mathrm{~kg} .{ }^{24}$ Our

342 results indicate that in American cocker spaniels the tympanic bulla volume is smaller in

343 relation to the body weight than what is reported previously in mesaticephalic dogs, but

344 there is variation between individuals. However, further studies with a larger number of

345 dogs are required to verify our results and to evaluate whether this anatomical variation has

346 any impact on the incidence of otitis in this breed.

Effusion in the bulla indicating likely otitis media was noted in 11 ears, the

348 majority in ears with clinically severe otitis externa. Mineralization of the ear canal and the

349 presence of cocci or rod-shaped bacteria in aural cytology were associated with tympanic

350 bulla abnormalities, while suppurative otitis was not, unlike in the study of Belmudes et al.

351 (2018)..$^{32}$ Differences in study populations are likely to explain these discrepancies. The study

352 of Belmudes et al. comprised dogs with chronic otitis externa, while majority of dogs in our 
study had clinically healthy ears. Middle ear effusion may also appear in dogs without a history or clinical findings of ear disease, especially in brachycephalic dog breeds. ${ }^{18,24-25}$ In such cases, the effusion may reflect accumulation of fluid unrelated to inflammation. ${ }^{18}$ For example trigeminal nerve disorder or mass may cause denervation of the tensor veli palatini muscle and dysfunction of tuba auditiva, leading to middle ear effusion. ${ }^{33,34}$ In one dog, the presence of bilateral bulla effusion was a surprising finding, because one ear showed only mild otitis externa and the other ear was considered clinically healthy, underlining the importance of diagnostic imaging in diagnosing middle ear abnormalities.

This study has some limitations. The number of dogs in each group was small, and female dogs were overrepresented in the American cocker spaniel group. The health status of the mesaticephalic dog group was based on retrospective data, hence it is possible that some of these dogs may have suffered from otitis externa previously. The different tympanic bulla measurements were performed by either one of the two observers, thus reliability or repeatability could not be measured. Ideally, bulla measurements should have been performed twice, but as some of the measurements were laborious, this was not 368 feasible. In conclusion, the horizontal ear canal of healthy American cocker spaniels was not narrower than that seen in similarly sized mesaticephalic dogs. The method of measuring

371 the transverse area of the horizontal ear canal appeared feasible with excellent intra- and 372 inter-observer repeatability. In American cocker spaniels, the mean tympanic bulla volume 373 was slightly smaller in relation to the body weight, and the tympanic bulla wall was thicker 374 than in previous reports for mesaticephalic dogs. Our results imply that breed-specific 375 differences in the anatomy of the bulla also appear in non-brachycephalic dog breeds. 
377 Author Contributions:

378 Category 1

379 (a) Conception and Design: Mirja Kaimio, Anu Lappalainen, Outi Laitinen-Vapaavuori

380 (b) Acquisition of Data: Mirja Kaimio, Anu Lappalainen

381 (c) Analysis and Interpretation of Data: Mirja Kaimio, Anu Lappalainen, Vahideh Rahmani,

382 Sofia Männikkö

383 Category 2

384 (a) Drafting the Article: Mirja Kaimio

385 (b) Revising Article for Intellectual Content: Anu Lappalainen, Vahideh Rahmani, Sofia

386 Männikkö, Outi Laitinen-Vapaavuori

387 Category 3

388 (a) Final Approval of the Completed Article: Mirja Kaimio, Anu Lappalainen, Vahideh

389 Rahmani, Sofia Männikkö, Outi Laitinen-Vapaavuori

390

391 Acknowledgements: The authors thank the Finnish Veterinary Research Foundation for

392 financial support and the American cocker spaniel kennel club for promoting the study.

393

394 References:

395 1. Baba E, Fukata T. Incidence of otitis externa in dogs and cats in Japan. Vet Rec

396 1981;108:393-395.

$3972 . \quad$ Carlotti D. Diagnosis and medical treatment of otitis externa in dogs and cats. J

398 Small Anim Pract 1991;32:394-400. 
400 otitis externa: a retrospective study of 100 cases. Vet Dermatol 2007;18:341-347.

401 4. Zur G, Lifshitz B, Bdolah-Abram T. The association between the signalment, 402 common causes of canine otitis externa and pathogens. J Small Anim Pract 2011;52:254-258.

403 5. Hill PB, Lo A, Eden CAN, Huntley S, Morey V, Ramsey S, Richardson C, Smith DJ, 404 Sutton C, Taylor MD, Thorpe E, Tidmarsh R, Williams V. Survey of the prevalence, diagnosis 405 and treatment of dermatological conditions in small animals in general practice. Vet Rec $406 \quad 2006 ; 158: 533-539$.

4076

Kaimio M, Saijonmaa-Koulumies L, Vapaavuori O. Surveys of otitis externa in

408 American cocker spaniels in Finland. Acta Vet Scand 2017;59:14. DOI 10.1186/s13028-017-

$409 \quad 0282-3$.

$4107 . \quad$ Mason LK, Harvey CE, Orsher RJ. Total ear canal ablation combined with lateral 411 bulla osteotomy for end-stage otitis in dogs. Vet Surgery 1988;17:263-268.

412 8. Matthiesen DT, Scavelli T. Total ear canal ablation and lateral bulla osteotomy 413 in 38 dogs. J Am Anim Hosp Assoc 1990;26:257-267.

$4149 . \quad$ White RAS, Pomeroy CJ. Total ear canal ablation and lateral bulla osteotomy in 415 the dog. J Small Anim Pract 1990;31:547-553.

416 10. Angus JC, Lichtensteiner C, Campbell KL, Schaeffer DJ. Breed variations in 417 histologic features of chronic severe otitis externa in dogs: 80 cases (1995-2001). J Am Vet 418 Med Assoc 2002;221:1000-1006.

419 11. ColeLK, KwochkaKW, KowalskiJJ, HillierA. Microbialfloraand antimicrobial 420 susceptibility patterns of isolated pathogens from the horizontal ear canal and middle ear in 421 dogs with otitis media. J Am Vet Med Assoc 1998;212:534-538. 

media in a dog. Vet Radiol Ultrasound 2000;41:46-49.

42614. Dickie AM, Doust R, Cromarty L, Johnson VS, Sullivan M, Boyd JS. Comparison

427 of ultrasonography, radiography and a single computed tomography slice for the

428 identification of fluid within the canine tympanic bulla. Res Vet Sci 2003;75:209-216.

429 15. Garosi LS, Dennis R, Schwarz T. Review of diagnostic imaging of ear disease in 430 the dog and cat. Vet Radiol Ultrasound 2003;44:137-146.

$431 \quad$ 16. Love NE, Kramer RW, Spodnick GJ, Thrall DE. Radiographic and computed 432 tomographic evaluation of otitis media in the dog. Vet Radiol Ultrasound 1995;36:375-379. 17. Rohleder JJ, Jones JC, Duncan RB, Larson MM, Waldron DL, Tromblee T.

434 Comparative performance of radiography and computed tomography in the diagnosis od 435 middle ear diseases in 31 dogs. Vet Radiol Ultrasound 2006;47:45-52.

436 18. Owen MC, Lamb CR, Lu D, Targett MP. Material on the middle ear of dogs 437 having magnetic resonance imaging for investigation of neurologic signs. Vet Radiol 438 Ultrasound 2004;45:149-155.

439 19. Doust R, King A, Hammond G, Cave T, Weinrauch S, Meelor D, Sullivan M. 440 Assessment of middle ear disease in the dog: a comparison of diagnostic modalities. J Small $441 \quad$ Anim Pract 2007;48:188-192.

442 20. Cole LK, Samii VF, Wagner SO, Rajala-Schultz PJ. Diagnosis of primary secretory 443 otitis media in the cavalier King Charles spaniel. Vet Dermatol 2015;26:459-466.

444 21. Smeak DD. Treatment of persistent deep infection after total ear canal ablation 445 and lateral bulla osteotomy. Vet Clin Small Anim 2016;46:609-621. 
447 tomographic anatomy of the canine inner and middle ear. Vet Radiol Ultrasound 2002;43:22-

44826.

449 23. Defalque VE, Rosenstein DS, Rosser EJ. Measurement of normal middle ear

450 cavity volume in mesaticephalic dogs. Vet Radiol Ultrasound 2005;46:490-493.

451 24. Salguero R, Herrtage M, Holmes M, Mannion P, Ladlow J. Comparison between

452 computed tomographic characteristics of the middle ear in nonbrachycephalic and

453 brachycephalic dogs with obstructive airway syndrome. Vet Radiol Ultrasound 2016;57:137-

454143.

455 25. Mielke B, Lam R, Ter Haar G. Computed tomographic morphometry of

456 tympanic bulla shape and position in brachycephalic and mesaticephalic dog breeds. Vet

457 Radiol Ultrasound 2017;58:552-558.

458 26. Seppänen RTK, Kaimio M, Schildt KJM, Lilja-Maula L, Hyytiäinen HK, Mölsä S,

459 Morelius M, Rajamäki MM, Lappalainen AK, Rantala M. Skin and ear health in a group of

460 English bulldogs in Finland - a descriptive study with special reference to owner perceptions.

$461 \quad$ Vet Dermatol 2019;30:307-e85.

462 27. Nuttal T, Bensignor E. A pilot study to develop an objective clinical score for

463 canine otitis externa. Vet Dermatol 2014;25:530-e92.

$464 \quad 28 . \quad$ Landis RJ, Koch GG. The measurement of observer agreement for categorial 465 data. Biometrics 1977;33:159-174.

$46629 . \quad$ Huang H-P, Little CJL, McNeil PE. Histological changes in the external ear canal

467 of dogs with otitis externa. Vet Dermatol 2009;20:422-428.

468 30. Cole LK, Podell M, Kwochka KW. Impedance audiometric measurements in 469 normal dogs. Am J Vet Res 2000;61:442-445. 
471 multidetector thin-slice computed tomography of the head. Vet Radiol Ultrasound

$472 \quad 2015 ; 56: 18-24$.

473 32. Belmudes A, Pressanti C, Barthez PY, Castilla-Costano E, Fabries L, Cadiergues

474 MC. Computed tomographic findings in 205 dogs with clinical signs compatible with middle

475 ear disease: a retrospective study. Vet Dermatol 2017;29:45-e20.

476 33. Kent M, Glass EN, de Lahunta A, Platt SR, Haley A. Prevalence of effusion in the 477 tympanic cavity in dogs with dysfunction of the trigeminal nerve: 18 cases (2004-2013). J Vet $478 \quad$ Intern Med 2013;27:1153-1158.

479 34. Wessmann A, Hennessey A, Goncalves R, Benigni L, Hammond G, Volk HA. The 480 association of middle ear effusion with trigeminal nerve mass lesions in dogs. Vet Record $481 \quad$ 2013;18:449. DOI 10.1136/vr.101817. 
Table 1. Otoscopic and cytological examination findings of the ears in American cocker

spaniels. A total OTIS3 score of $\geq 4$ differentiated affected from clinically healthy ears.

\begin{tabular}{|r|c|c|c|}
\hline Erythema & $\begin{array}{c}\text { Clinically healthy } \\
\text { ears } \\
(n=40)\end{array}$ & $\begin{array}{c}\text { Clinically mild } \\
\text { otitis externa } \\
(n=13)\end{array}$ & $\begin{array}{c}\text { Clinically severe } \\
\text { otitis externa } \\
(n=22)\end{array}$ \\
\hline $\begin{array}{r}\text { OTIS3 score for different } \\
\text { parameters: mean } \pm \text { SD (range) }\end{array}$ & $\begin{array}{c}0.3 \pm 0.4 \\
(0-1)\end{array}$ & $\begin{array}{c}0.6 \pm 0.5 \\
(0-1)\end{array}$ & $\begin{array}{c}1.2 \pm 0.5 \\
(1-3)\end{array}$ \\
\hline Edema & $\begin{array}{c}0.5 \pm 0.6 \\
(0-2)\end{array}$ & $\begin{array}{c}0.9 \pm 0.8 \\
(0-2)\end{array}$ & $\begin{array}{c}2.8 \pm 0.4 \\
(2-3)\end{array}$ \\
\hline Exudate & $0.6 \pm 0.5$ & $2.5 \pm 0.7$ & $2.3 \pm 0.7$ \\
$(0-2)$ & $(1-3)$ & 0 \\
\hline Ulceration & 0 & 0 & $6.4 \pm 0.6$ \\
Total & $1.3 \pm 1.2$ & $4.2 \pm 0.4$ & $(6-8)$ \\
\hline $\begin{array}{r}\text { Number of ears with the following } \\
\text { cytological findings: }\end{array}$ & $(0-3)$ & $(4-5)$ & 10 \\
\hline Malassezia spp. & 17 & & 14 \\
\hline Cocci & 1 & 12 & 16 \\
\hline Rods & 0 & 1 & 5 \\
\hline Inflammatory cells & 0 & 1 & \\
\hline & & 1 & $0.3)$ \\
\hline
\end{tabular}

496

OTIS3 scoring: The presence and amount of erythema, edema, exudate and

erosion/ulceration in the ear canal were scored with 0-3 scale as follows: none (score 0), 

cocker spaniels and mesaticephalic dogs in the study.

\begin{tabular}{|c|c|c|c|}
\hline & $\begin{array}{c}\text { American cocker spaniels } \\
(\mathrm{n}=9,18 \text { ears })\end{array}$ & $\begin{array}{c}\text { Mesaticephalic dogs } \\
(\mathrm{n}=23,46 \text { ears })\end{array}$ & $P$-value \\
\hline Weight (kg) & $10.0 \pm 0.9(8.3-11.5)$ & $10.4 \pm 4.7(5.2-19.5)$ & 0.562 \\
\hline Age (years) & $4.3 \pm 2.2(1.2-8.0)$ & $7.1 \pm 3.7(1.8-13.0)$ & $0.042^{*}$ \\
\hline Skull size (cm) & $10.5 \pm 0.4(9.8-11.0)$ & $10.4 \pm 1.2(8.2-12.7)$ & 0.552 \\
\hline TA1 $\left(\mathrm{mm}^{2}\right)$ & $27.2 \pm 1.6(24.6-30.1)$ & $29.2 \pm 7.0(12.3-45.0)$ & 0.522 \\
\hline $\begin{array}{c}\text { TA2 } \\
\left(\mathrm{mm}^{2}\right)\end{array}$ & $37.1 \pm 7.8(24.6-51.4)$ & $35.6 \pm 10.1(16.9-59.4)$ & 0.247 \\
\hline
\end{tabular}

509

SD = Standard Deviation

511 TA1 $=$ Transverse area $\left(\mathrm{mm}^{2}\right)$ of the osseous part of the horizontal ear canal

512 TA2 $=$ Transverse area $\left(\mathrm{mm}^{2}\right)$ of the widest air-filled part of the horizontal ear canal

$513 *$ = significant difference between the two groups

514

515

516 
Table 3. Tympanic bulla measurements in American cocker spaniels.

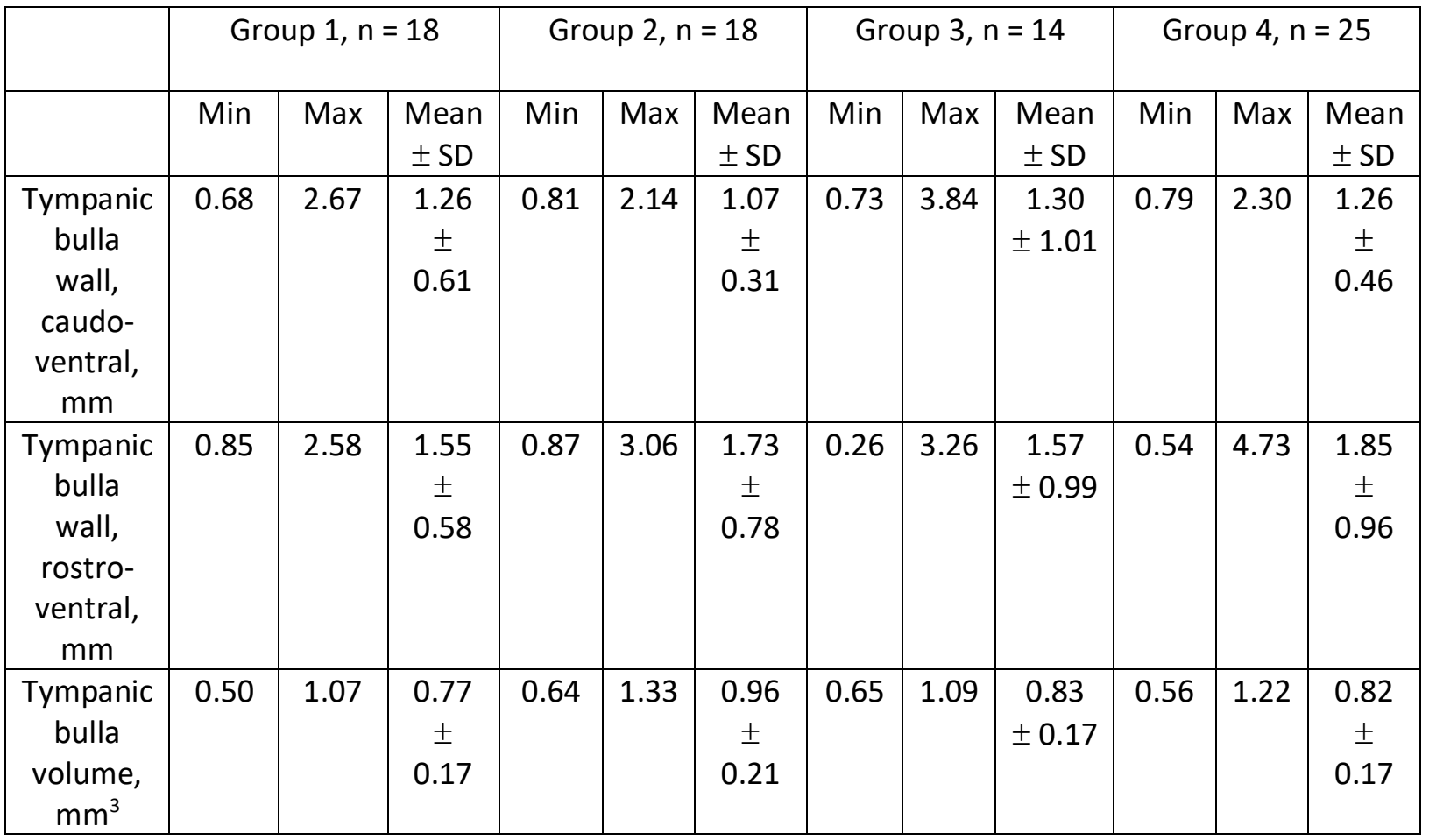

SD: Standard Deviation

526 Group 1: Dogs with clinically healthy ears and no history of ear disease.

527 Group 2: Dogs with clinically healthy ears, and a history of ear disease.

528 Group 3: Dogs with clinically mild otitis externa.

529 Group 4: Dogs with clinically severe otitis externa. 
Figure legends:

539 Figure 1A-C: Multiplanar reconstruction images (middle-ear algorithm, window level $700 \mathrm{HU}$, 540 window width $4000 \mathrm{HU}$ ) showing the measurement point for measuring the transverse area $541\left(\mathrm{~mm}^{2}\right)$ of the osseous (TA1) (B) and widest air-filled part of the cartilaginous (TA2) (C)

542 horizontal ear canal.

543 Figure 2: The transverse area $\left(\mathrm{mm}^{2}\right)$ of the horizontal ear canal was determined by manually

544 drawing a region of interest with the software's pencil tool and tracing the margin of the

545 bony wall of the osseous canal (A) or the air-filled cartilaginous canal (B).

546 Figures 3: The thickness of the tympanic bulla wall $(\mathrm{mm})$ was measured in the transverse 547 plane images (middle-ear algorithm, window level $700 \mathrm{HU}$, window width $4000 \mathrm{HU}$ ) at the 548 level of the tympanic membrane (caudo-ventral aspect) (A) and at the level of the cochlea 549 (rostro-ventral aspect) (B).

$550 \quad$ Figure 4: The skull width and height $(\mathrm{cm})$ were measured from the transverse plane images 551 (middle-ear algorithm, window level $700 \mathrm{HU}$, window width $4000 \mathrm{HU}$ ) at the level of the 552 tympanic membrane as shown in the images.

553 Figure 5: Comparison of the transverse area $\left(\mathrm{mm}^{2}\right)$ of the osseous part of the horizontal ear 554 canal (TA1) in different American cocker spaniel groups. No significant differences were 555 detected between the groups. Group 1: dogs with clinically healthy ears and no history of 556 ear disease. Group 2: dogs with clinically healthy ears, and a history of ear disease. Group 3: 557 dogs with clinically mild otitis externa. Group 4: dogs with clinically severe otitis externa. The 558 whiskers of the box plot show the minimum and maximum of the data, lower line of the box 559 presents the lower quartile, upper line the upper quartile and the line in the middle the 560 median. Outliers are presented with circles. 
561 Figure 6: Comparison of the transverse area $\left(\mathrm{mm}^{2}\right)$ of the widest air-filled part of the

562 cartilaginous horizontal ear canal (TA2) in different American cocker spaniel groups. The TA2

563 values in group 4 were significantly smaller than in the other groups. Group 1: Dogs with

564 clinically healthy ears and no history of ear disease. Group 2: Dogs with clinically healthy

565 ears, and a history of ear disease. Group 3: Dogs with clinically mild otitis externa. Group 4:

566 Dogs with clinically severe otitis externa. The whiskers of the box plot show the minimum

567 and maximum of the data, lower line of the box presents the lower quartile, upper line the

568 upper quartile and the line in the middle the median. Outliers are presented with circles.

569 Figure 7: The thickness of the tympanic bulla wall varied between individuals. These images

570 (slice thickness $1 \mathrm{~mm}$, bone algorithm, window level $500 \mathrm{HU}$, window width $3500 \mathrm{HU}$ ) are

571 from a clinically healthy American cocker spaniel with no history of ear disease, but with a

572 thick tympanic bulla wall. The thickness of the tympanic bulla wall was measured at the level

573 of the tympanic membrane (A) and at the level of the cochlea (B). Compare with Figure 3.

574 Figure 8: The Bland-Altman plot presents the difference between the calculated (MECV) and

575 the measured tympanic bulla volume values in American cocker spaniels against the mean of

576 these two. MECV $=-0.612+0.757 * \ln$ (bodyweight) 


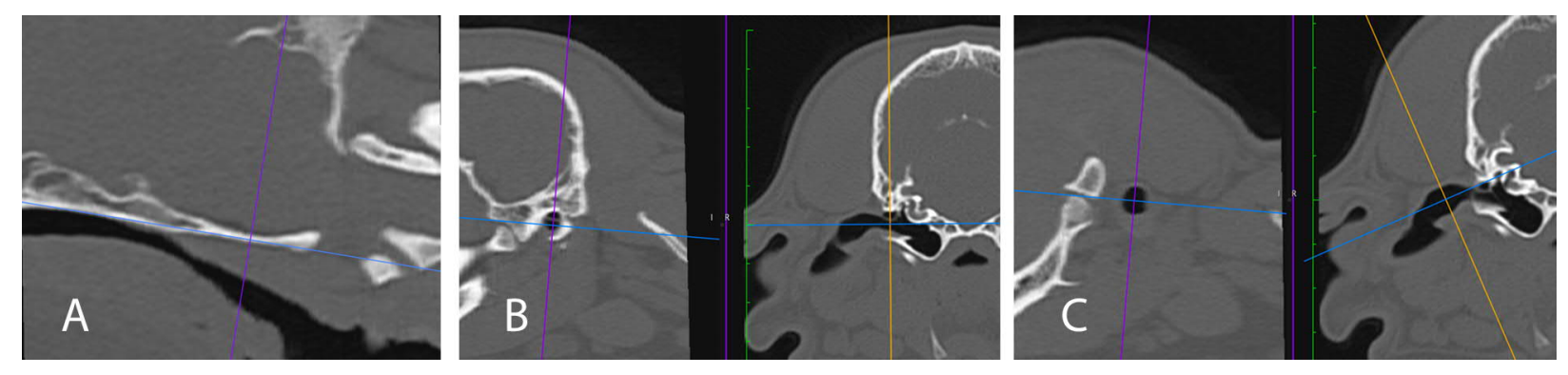




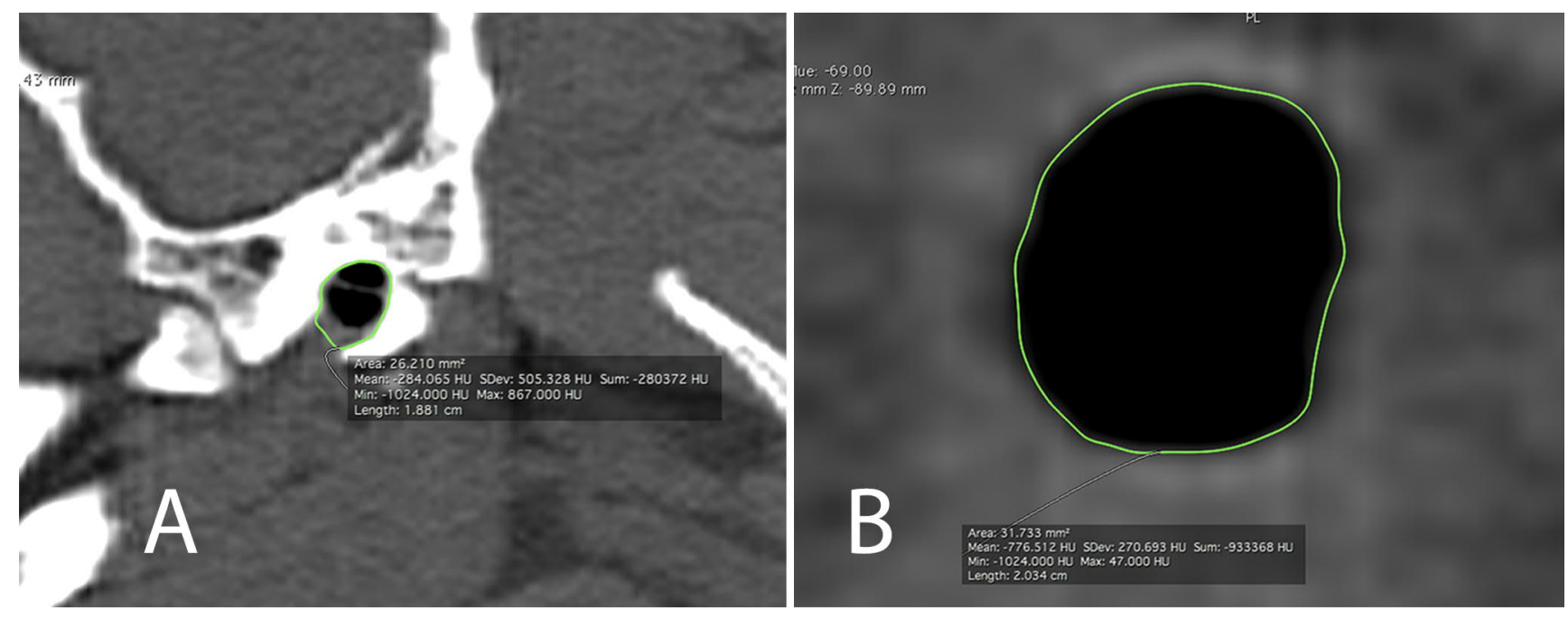



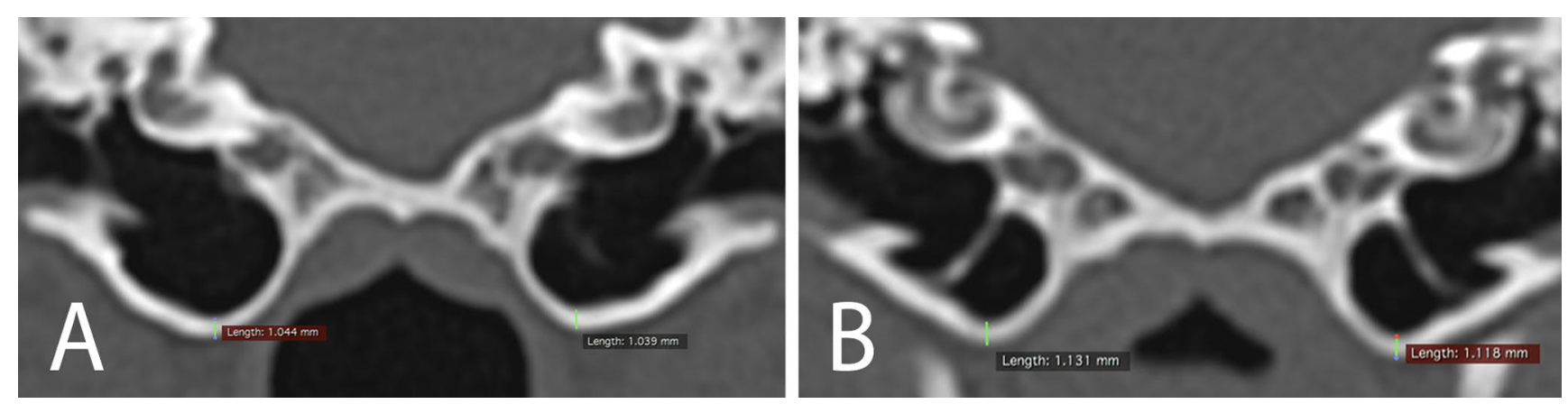


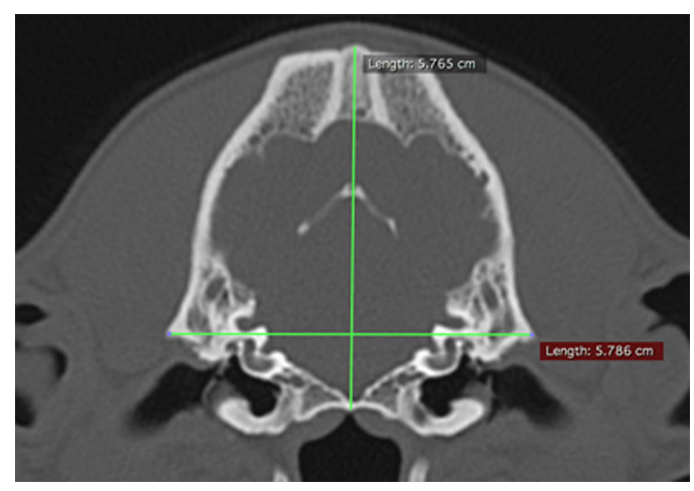




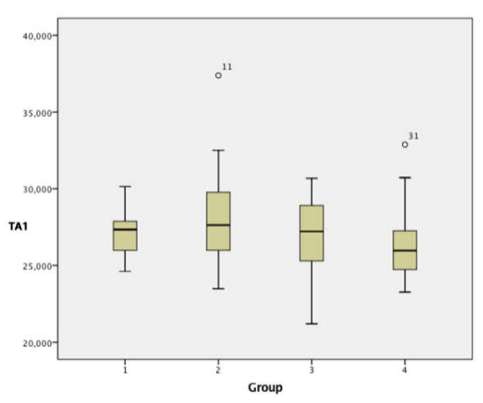




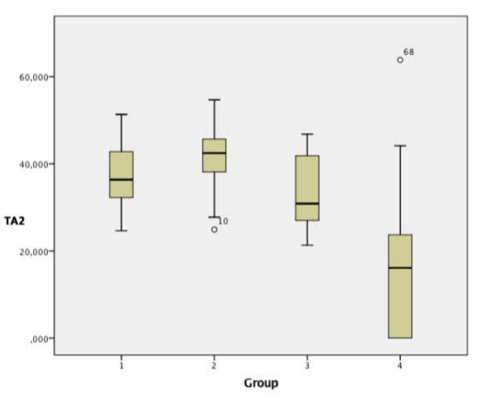



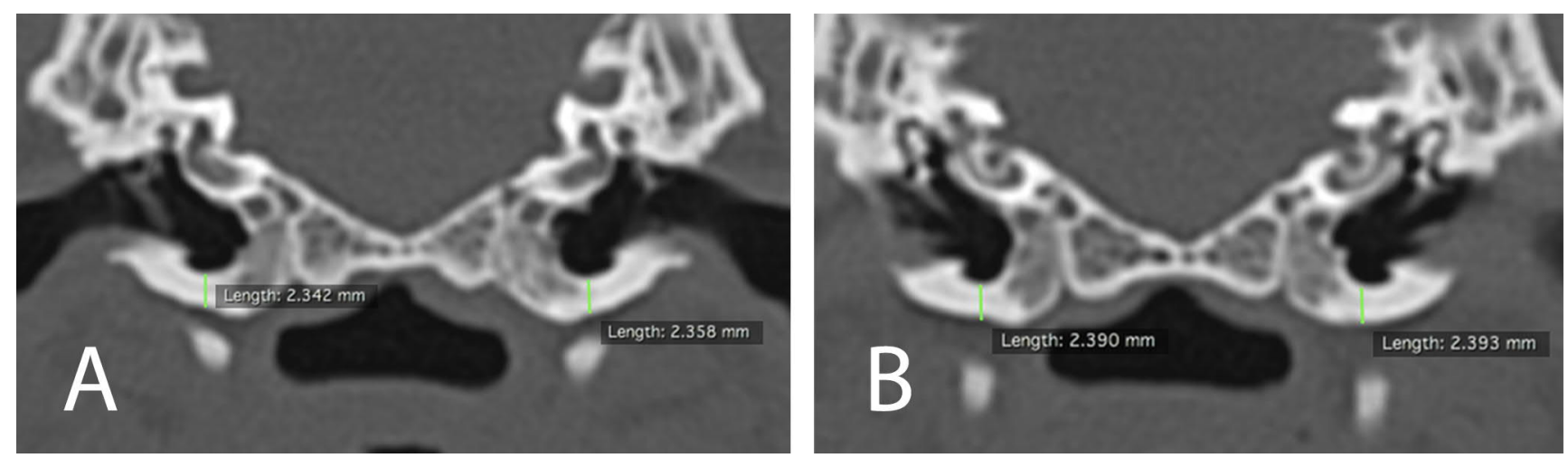


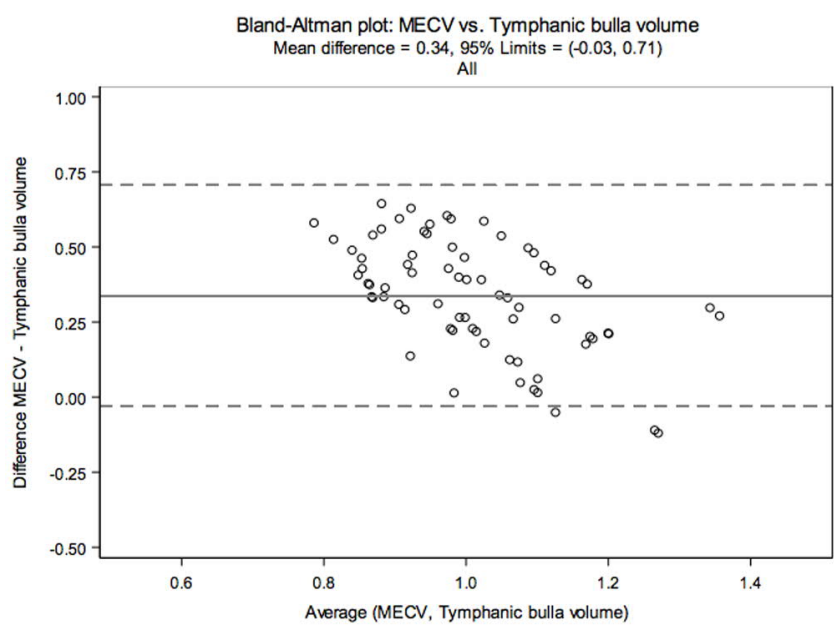

